\title{
Denoising of Fetal Phonocardiogram Signal by Wavelet Transformation
}

\author{
Irmalia Suryani Faradisa ${ }^{1,2, *}$, Ananda Ananda $^{3}$, Tri Arief Sardjono ${ }^{1}$, and \\ Mauridhi Hery Purnomo ${ }^{1}$ \\ ${ }^{1}$ Electrical Engineering Department, Institut Teknologi Sepuluh Nopember, Jl. Teknik Kimia, \\ Surabaya 60111, East Java, Indonesia \\ ${ }^{2}$ Electrical Engineering Department, Institut Teknologi Nasional Malang, Jl. Sigura - Gura No.2, \\ Malang 65152, East Java, Indonesia \\ ${ }^{3}$ School of Mathematics, Computer Science and Engineering, City, University of London, \\ Northampton Square, Clerkenwell, London EC1V 0HB, United Kingdom
}

\begin{abstract}
Auscultation is still one of the most basic analytical tools used to determine the fetal heart's functional state as well as the first fetal wellbeing measure. It is called fetal phonocardiography (fPCG) in its modern form. The technique of fPCG is passive and can be used to track long-term. Robust signal processing techniques are required to denoise the signals in order to improve the diagnostic capabilities of fPCG. A linear filter is used to eliminate distortion and interference from the fPCG signals through conventional denoising techniques. This paper searched for optimal configuration of the wavelet based denoising system. Based on the experimental results, can be conclude that the signal should be decomposed on six levels. From this it can be seen that the lowest MSE (mean square error) value is the use of coiflets three with SURE threshold algorithm with hard threshold parameters.
\end{abstract}

Keywords: Auscultation, decomposition, fetal heart sounds, threshold, mean square error.

\section{Introduction}

One of the key factors to focus on in terms of tracking a fetus ' well-being has always been fetal heart sounds. In the past, the main procedure during midwifery and obstetrics was sporadic auscultation, and the clinicians ' main equipment was Pinard Horn. The method's reliability depended heavily on the examiner's expertise and experiences [1]. This approach was eventually replaced by the continuous Electronic Fetal Monitoring (EFM), also known as Cardiotocography (CTG), using the Doppler effect of fetal heart rate monitoring (fHR). The method's output should be better than sporadic auscultation by using computer technology. Several studies, however, suggest this inference is doubtfull [2]. In contrast, the downside of Doppler-based EFM is that it is not capable of measuring the variability of fetal heart rate. Many short-term changes may therefore occur unnoticed [2].

The recording of fetal heart sounds has been resurrected in the fetal phonocardiogram (fPCG) over the past few years. Compared to sporadic auscultation, this approach allows

\footnotetext{
*Corresponding author: irmalia15@mhs.ee.its.ac.id
} 
the digitization of heart sounds and therefore more accurate assessment and analysis based on computers [3]. In addition, unlike the CTG, it makes it possible to measure the variability of the heart rate and to detect certain additional features of the fPCG signal (e.g.sub audible noises, murmurs, etc.). This approach therefore has great potential to enhance fetal monitoring performance. However, it suffers from the interference with the desired signal being detected. Current denoising approaches that use linear filters to eliminate fPCG noise face some limitations due to the fPCG signals non-stationarity. As a result, most recent studies concentrate on rejecting fPCG signals using advanced signal processing methods to enhance the diagnostic capabilities of this method [4-6].

In this analysis, for denoising the abdominal fPCG data, the discrete wavelet transformation was applied. Some researchers have proposed various wavelet-based fPCG filtration system approaches and settings [7-9]. There are three key parameters to be carefully selected, namely wavelet base, threshold form, and decomposition rate. In choosing these parameters, most of the published works $[7,8,10]$ present heuristic approaches. Experimental aspect of this analysis presents an analytical technique of optimization that can help to determine the validity of the variable for the particular purpose.

Experiments on synthetic PCG and real stomach data are performed. Synthetic information is used to refine and test denosis systems. The first step consists of optimizing the parameters of the system: wavelet families and the degree of decomposition. The orthogonal members (sym, db, coif) were examined for six decomposition rates. In the second phase, the comparison of the thresholding approach used conventionally is included in the Matlab Wavelet Toolbox, namely SURE, Heuristic SURE and Minimax.

\section{Fetal phonocardiographic signal}

Fetal phonocardiography is the recording from the maternal abdominal layer of natural vibroacoustic signals. The fPCG signal provides valuable information about unborn anatomy [9]. It is also able to recognize additional potential fetal heart abnormal signals such as those related to heart murmur, split effect, and breathing changes that can be identified from abdominal sound (fPCG) signals study. Nevertheless, because of its operating theory, these cardiac irregularities can not be identified with the widely accepted fCTG procedure [9]. Phonocardiography also offers a reliable and enduring record of fetal heart sounds that can prove to be of great prognostic value through contrast at a later stage. Biomedical scientists and medical experts have developed the fPCG technique earlier but ignored primarily because of its poo $r$ signal-to-noise ratio (SNR) [11].

It is a linear summation of the fPCG signals [9]:

i. Fetal heart sound;

ii. Internal noise;

iii. External noise.

The fetal heartbeat is a signal created by the mechanical action of the fetal heart. The fetal heart is basically split into two chamber pairs and has four valves: the mitral and the tricuspid valves. In the fetal cardiac cycle, the blood attempts to flow back into the lower pressure atrial chambers when the ventricles start to contract. This reverse blood flow is halted by shutting down the mitral and tricuspid valves, which produce the first heart signal (S1). Whenever the pressure in the ventricular chambers becomes too high to tolerate the pulmonary valves, they open and the blood pressure is quickly thrown into the arteries. While the ventricles are being drained, there is a reduction in the pressure of the remaining blood in the arteries. This gradient in pressure allows the blood of the heart to return to the ventricles. The reverse flow is halted by the pulmonary valves by closing, resulting in the second heart tone (S2) [11]. The fetal heart sound frequency range is below $200 \mathrm{~Hz}$. 
Internal noise is a spontaneous signal triggered by maternal breathing noises, fetal movement auditory noise, maternal digestive tone, maternal breathing rhythm, maternal heart sound, and placental blood turbulence. For key frequency components from $0 \mathrm{~Hz}$ to $25 \mathrm{~Hz}$ [6], these noises are of low amplitude.

Likewise, internal noise is a mixture of shear noise from sensor motion during recording and ambient noise from the atmosphere such as fan vibration, air conditioner, and surrounding people's hue and cry. It is of high amplitude and frequency (100 Hz to $20000 \mathrm{~Hz}$ ) in contrast [6].

\section{Material}

The data used in this paper were secondary data. The root of a server is Shiraz University's (SUFHSDB) fetal heart database [12]. This database was built using recordings of 109 pregnant women (mothers aged $16 \mathrm{yr}$ to $47 \mathrm{yr}$, mean \pm SD: $29.3 \mathrm{yr} \pm 5.8 \mathrm{yr}$ with BMI 19.5 to 38.9 mean $\pm \mathrm{SD}: 29.2 \pm 4.0$ ), with a digital JABES electronic stethoscope (GS Technology Co. Ltd., South Korea) positioned on the lower maternal abdomen as defined in (Samieinasab and Sameni 2015).

The data was collected twice in the case of twins (seven cases) according to the locations recommended by the gynecologist expert. For capturing and manipulating signals on a Mac, Audacity ${ }^{\circledR}$ cross-platform audio technology was used. A maximum of 99 subjects had recorded one signal, three subjects had reported two and seven individual cases of twins, resulting in 119 total recordings. Can record's average duration is about $90 \mathrm{~s}$. Generally speaking, the sampling frequency was $16000 \mathrm{~Hz}$ with 16-bit quantization and some $44100 \mathrm{~Hz}$ recordings. The data was collected in digital stethoscope wide-band mode with a $20 \mathrm{~Hz}$ to $1 \mathrm{kHz}$ frequency response.

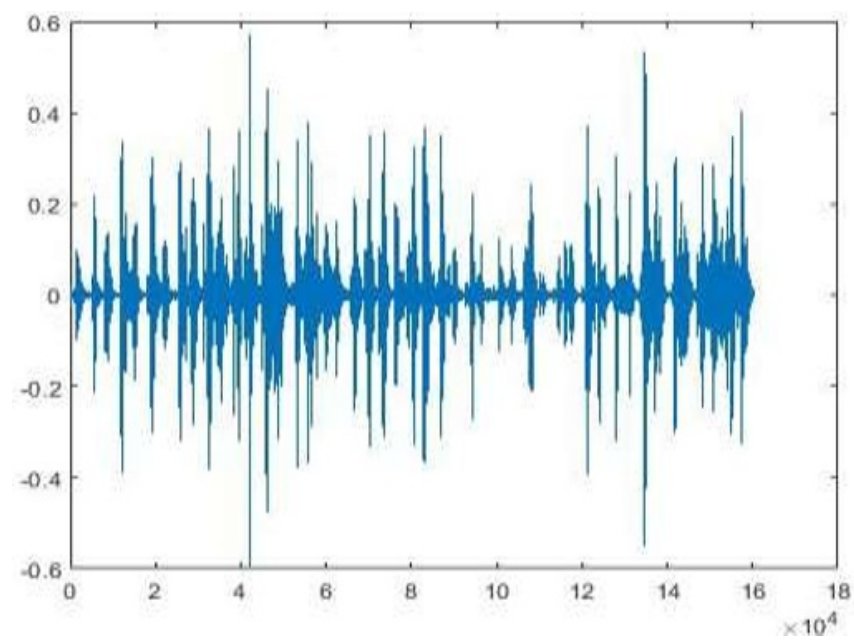

Fig. 1. Typical fPCG signal.

\section{Result}

\subsection{Wavelet Decomposition}

WT (Wavelet Decomposition) is a two-dimensional timescale processing method for nonstationary signals with sufficient scale and time shift values. Through dilating and 
compressing its basic functions, it is capable of representing signals in different resolutions. The WT's main advantage is that it has different window sizes, is broad at low frequencies and narrow at high frequencies, resulting in maximum time-frequency resolution in all domain ranges [13].

Using DWT, the fPCG signal is broken down into a frequency representation. The DWT's main advantage is that it provides good resolution of the time. The DWT will reveal the local characteristics of the input signal due to its great time and frequency position capacity. Tests are carried out of mother wavelets: Symlets wavelet, Daubechies wavelet and Coiflets wavelet. The best results will be used as a mother wavelet. Maternal wavelet selection is based on the similarities between the PPCG signal and the maternal wavelet signal form. For this reason, coiflet 3, sym5, db6 were chosen.

Table 1. Table selection of mother wavelet and decomposition level.

\begin{tabular}{|c|c|c|c|}
\hline \multirow{2}{*}{ Level } & \multicolumn{3}{|c|}{ Wavelet Family } \\
\cline { 2 - 4 } & Coiflet 3 & Sym5 & Db6 \\
\hline 1 & 0.0725 & 0.0725 & 0.0725 \\
\hline 2 & 0.0710 & 0.0710 & 0.0710 \\
\hline 3 & 0.0710 & 0.0710 & 0.0710 \\
\hline 4 & 0.0710 & 0.0710 & 0.0710 \\
\hline 5 & 0.0395 & 0.0392 & 0.0390 \\
\hline 6 & 0.0280 & 0.0283 & 0.0281 \\
\hline
\end{tabular}

Table 1 consists of optimizing wavelet family system parameters and level decomposition. Sym5, db6, coiflet 3 were tested for six levels of decomposition.
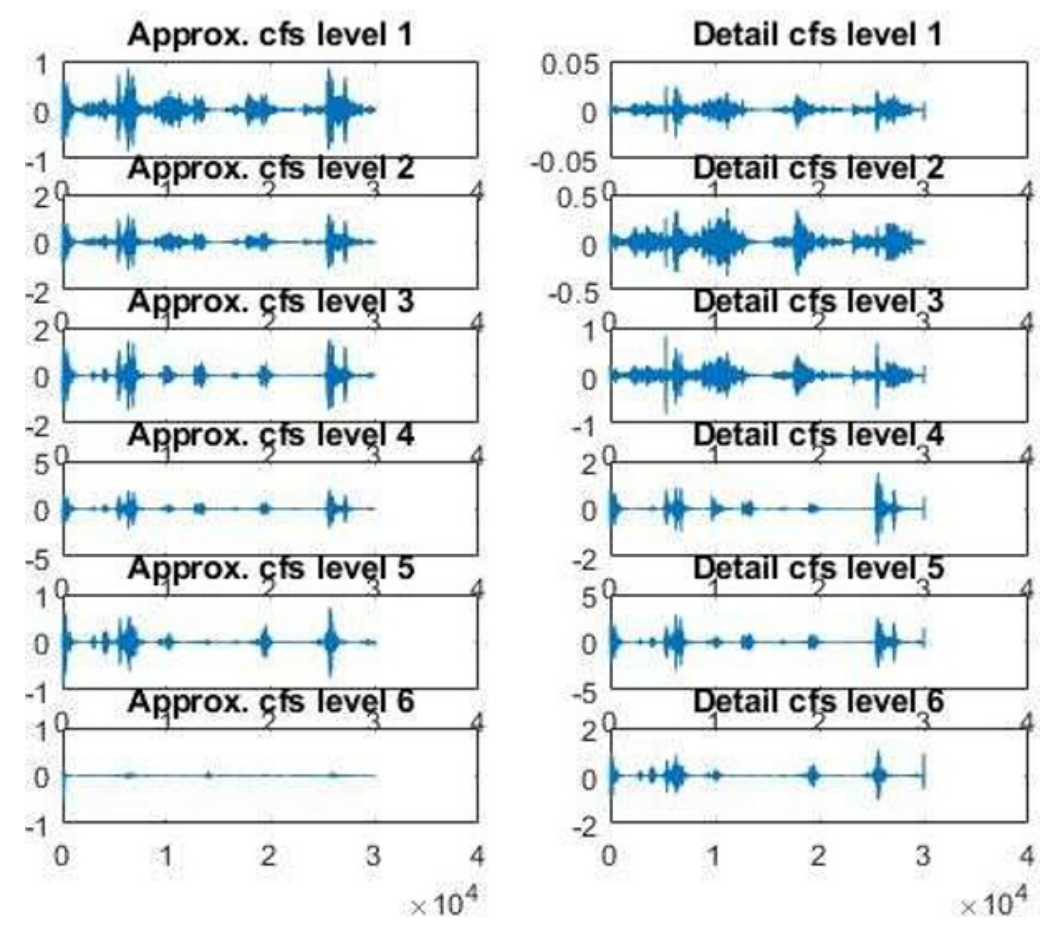

Fig 2. Coefficients of the wavelet decomposition.

Figure 2 is a fPCG signal that has been through a decomposition process that is carried out using a mother wavelet coiflet 3 for six level decomposition. 


\subsection{Denoising}

The noise can be further extracted by wavelet denoising technique after the wavelet decomposition of the reported signals. This method preserves the signal that includes the coefficients of decomposition and eliminates those connected to the noise. Choosing the threshold level that is, deciding the noise and signal dividing point will have a major impact on the denoising effect. If the threshold level is too low, too much noise will be allocated, on the contrary, some signal data will be lost if the threshold level is too high. The two common methods of tresholding a signal are:

i. Soft thresolding;

ii. Hard tresholding

The denoising algorithm uses linear regression of noisy parameters over time to achieve a non-parametric approximation of the reconstructed signal without noise. The widely used threshold algorithms for denosing non-stationary signals are:

i. Universal threshold (sqtwolog);

ii. Minimax threshold (minimaxi);

iii. Rigorous Stein's Unbiased Risk Estimate (SURE) threshold (Rigrsure).

Table 2. Table Comparison MSE of denoising algorithm

\begin{tabular}{|c|c|c|c|c|c|c|}
\hline & \multicolumn{2}{|c|}{ SURE } & \multicolumn{2}{c|}{ Minimax } & \multicolumn{2}{c|}{ Sqtw } \\
\cline { 2 - 7 } & $(\mathbf{s})$ & $(\mathbf{h})$ & $(\mathbf{s})$ & $(\mathbf{h})$ & $(\mathbf{s})$ & $(\mathbf{h})$ \\
\hline Sym5 & 0.000308 & 0.000227 & 0.000380 & 0.000325 & 0.000400 & 0.000400 \\
\hline Db6 & 0.000324 & 0.000303 & 0.000356 & 0.000279 & 0.000376 & 0.000376 \\
\hline coif3 & 0.000204 & 0.000150 & 0.000326 & 0.000329 & 0.000324 & 0.000322 \\
\hline
\end{tabular}

From this it can be seen that the lowest MSE (mean sqaure Error) value is the use of coiflets 3 with SURE threshold algorithm with hard threshold parameters.

\subsection{Wavelet reconstruction}

The reverse process of decomposition and analysis is reconstruction or synthesis. The decomposed coefficients are assembled back into the original signal in reconstruction without loss of information.

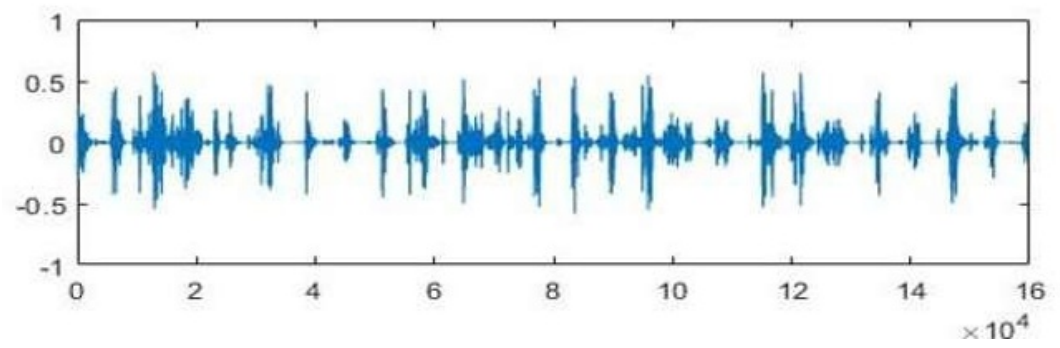

Fig 3. Recontruction version of the fPCG sign.

\section{Conclusion}

Phonocardiography is an appropriate tool for monitoring fetal well-being, which has been studied for years by many research labs, mostly because of its passive existence. The main benefit of this technique is its long-term measurement capacity, which is necessary due to the high varibilitas of the parameters of fetal cardiac activity. This paper searched for optimal wavelet-based denoising system configuration in this paper. It can be conclude that 
the signal should be decomposed at six levels based on the experimental results. From this it can be seen that using coiflets 3 with SURE threshold algorithm with hard threshold parameters is the lowest MSE (mean square error).

The research reported in this publication is supported by a BPPDN scholarship (No. contract: 1265.5/E4.4/2015) from the Ministry of Education of the Republic of Indonesia and the National Institute of Technology, Malang, Indonesia.

\section{Reference}

1. D. Lewis, S. Downe. Int. J. Gynecol. Obstet., 131,1:9-12(2015). https://obgyn.onlinelibrary.wiley.com/doi/full/10.1016/j.ijgo.2015.06.019

2. R. Kahankova, R. Martinek. Comparasion of fetal phonocardiogram wavelet denoising methods. Paper Presented in Signal Processing in Medicine and Biology Symposium (SPMB) (Pennsylvania, United Stated, 2018). https://ieeexplore.ieee.org/document/8615621

3. J. Kolarik, M. Golembiovsky, T. Docekal, R. Kahankova, R. Martinek, M. Prauzek. IFAC-PapersOnLine, 51,6:426-431(2018). https://www.sciencedirect.com/science/article/pii/S2405896318308607

4. R. Kahankova, R. Martinek, R. Jaros, J. Nedoma, M. Fajkus, J. Vanus. IFACPapersOnLine, 51,6:60-65(2018). https://www.sciencedirect.com/science/article/pii/S2405896318308747

5. A.S. Bureev, D.S. Zhdanov, N.N. Zilberman, E.Y. Kiseleva, S.Y. Yuriev. Biosci. Biotech. Res. Asia, 12,2:1743-1750(2015).

https://www.biotech-asia.org/vol12no2/comparative-assessment-of-24-hour-fetalmonitoring-methods-based-on-cardiac-rhythm/

6. F. KováCs, C. HorváTh, A.T. Balogh, G. Hosszú. Comput. Meth. Prog. Bio., 104,1:1925(2011). https://www.sciencedirect.com/science/article/abs/pii/S0169260710002683?via\%3Dihu $\underline{\mathrm{b}}$

7. A. Misal, G.R. Sinha. Advances in Computational Research, 4,1:46-49(2012). http://www.oalib.com/paper/2349534

8. S. Vaisman, S.Y. Salem, G. Holcberg, A.B. Geva. Comput. Biol. Med., 42,2:171179(2012). https://www.ncbi.nlm.nih.gov/pubmed/22169397

9. V.S. Chourasia, A.K. Tiwari. Crit. Rev. Biomed. Eng., 36(5-6):335-373(2008). https://www.ncbi.nlm.nih.gov/pubmed/20092429

10. V.S. Chourasia, A.K.J. Mittra, Med. Eng. Technol., 33,6:442-448(2009). https://www.ncbi.nlm.nih.gov/pubmed/19484684

11. M. Ruffo, M. Cesarelli, M. Romano, P. Bifulco, A. Fratini. Biomed. Signal Proces. 5,2:131-141(2010). https://www.sciencedirect.com/science/article/abs/pii/S1746809410000078?via\%3Dihu $\underline{b}$

12. R. Sameni. Shiraz University's (SUFHSDB) fetal heart database [Online] from https://physionet.org/content/sufhsdb/1.0.0/ (2017). [Accessed on August 20, 2020]. 
13. A. Ordaz-Moreno, R.J. Romero-Troncoso, J.A. Vite-Frias. Hardware signal processing unit for one dimensional variable length discrete wavelet transform. Paper Presented in The International Conference of The IEEE on Reconfigurable Computing and FPGAs, (Puebla City, Mexico, 2005). https://ieeexplore.ieee.org/document/1592487 